\title{
Implementation of Health Monitoring System Internet of Things
}

\author{
Kamalakkannan.S ${ }^{1}$, Mohanraj $V^{2}$, Naven $R^{3}$ Sam peter $S^{4}$ \\ Sri Krishna College of Technology, Coimbatore ${ }^{1}$ \\ Assistant Professor, Computer Science and Engineering, Sri Krishna college of Technology, Coimbatore ${ }^{2}$
}

\begin{abstract}
The Internet of Things is an growing topic of technical, social, and economic significance. IOT involves in various departments like Medical industries, Automobile industries, Manufacturing industries, and etc. Now a day's utility components everyday objects are being combined with Internet connectivity and powerful data analytic capabilities that promise to transform the way of life style. In this project a for comma patient a Dynamic Service Non Dependency Verification has been implemented using IOT. The first process of this project initiate with hardware interface. And we propose a heterogeneous IOT scheme to secure communication between a sensor node and an Internet host. We prove that this scheme is indistinguishable against various conditions. This project has the following advantages. First, it achieves confidentiality, integrity and authentication in a logical single step. Second, it allows a sensor node in an identity-based Enhanced data transfer to send a message to an Internet host in a public key infrastructure method.
\end{abstract}

Keywords: Internet of Things, Dynamic Service Non Dependency Verification.

\section{INTRODUCTION}

This project is entitles as "Implementing Internet of things in Health Monitoring for Comma Patients" is developed using IOT hardware kit as the transmitter and cloud sever as receiver. Hyper terminal tool has been used for PC interface. The main objective of this project is to develop a web based application to communication with an internet server in a secured manner for comma patients. The Internet of Things is an growing topic of technical, social, and economic significance. IOT involves in various departments like Medical industries, Automobile industries, Manufacturing industries, and etc. Now a day's utility components everyday objects are being combined with Internet connectivity and powerful data analytic capabilities that promise to transform life style. The term Internet of Things generally refers to scenarios where network connectivity and computing extends to objects, sensors and objects for everyday usage, allowing these devices to generate exchange and consume data with minimized human interaction. In this project a for comma patient a Dynamic Service Non Dependency Verification has been implemented using IOT. The first process of this project initiate with hardware interface. The hardware has been designed using 8051 microprocessor. The microprocessor contains 40 pin. More interactions can be done using 8051 pin interaction. In added with various sensors can be connected through the controller board interface. Multi sensors have been interacted in this hardware.

The hardware interactions are follows.

-Heart beat sensor - Checks heartbeat and Warns if abnormal

- Flux Sensor - Find fine movements in body

- Thermostat Sensor - Find body temperature

-Arduino Microcontroller - Main interface board

- COMM to USB convertor - For System interface

All the above mentioned hardware process has been implemented in a single interface controller board. All the sensors will be wired over the body of the comma patients. The sensors value will be uploaded in a centralized cloud server. A threshold value will be assigned for each sensor. In case of any abnormal means, the warns immediately to the user interface end. Secure Service Virtualization in IoT by Dynamic Service Non Dependency Verification is ensured by the hardware phase initially.

In previous work, the system collects the physical information from the patient who wears the wearable medical sensors and transmits it to the cloud. The output is displayed by using the mobile applications and a website that is hosted. The same sensors used as previous system are Blood pressure monitor. 


\section{IJARCCE}

\section{International Journal of Advanced Research in Computer and Communication Engineering}

Vol. 8, Issue 2, February 2019

The different sensors used are

1. Flux sensor-to find the movements in the body 2. Temperature sensor- to find the temperature of the body 3 . Heart beat sensor-to find the heart beat rate of the patient.

However, we have some drawbacks in existing process, there are, cannot find the movements in the body, the normal people cannot understand the output variations, there is no data visualisation techniques are used to predict the progress of the patient.

\section{LITERATURE REVIEW}

This section provides the basic significance of workflow scheduling in cloud. It also provides the numerous methodology. This development has resulted in huge usage of many applications.

The Internet of Things: A survey L. Atzori, A. Iera, and G. Morabito, 2010- This paper presents Internet of Things in a wider context. Main enabling factor of this concept is to integrate different type of technologies. In this paper, we describe the key technologies involved in the implementation of Internet of Things and the major application domain where the Internet of Things will play a vital role. Later we will discuss about the issues which are to be addressed before the worldwide acceptance of these technologies. There are lots of open issues to address.

Real-time location and patient monitoring system based on passive RFID P. Najera, J. Lopez, and R. Roman, 2011-. This study is about the literature on RFID applications in healthcare based on a formal research framework. We aim to identify opportunities, potential benefits and adoption barriers. Our study shows that most care providers indicated that RFID to be functional and useful in asset tracking and patient identification. Major barriers to RFID adoption in healthcare include prohibitive costs, technological limitations, and privacy concerns. Although RFID offers healthcare practitioners advantages to enhance clinical practice, better designed RFID systems are needed to increase acceptance and proper use of RFID in healthcare.

The adoption and implementation of RFID technologies in healthcare: A literature review, W. Yao, C. Chu, and Z. Li, 2012- In this paper, we develop a conceptual framework that can be used to classify publications on RFID applications and issues in the healthcare industry. We then use this framework to systematically summarize relevant articles. We conclude by highlighting future research directions where the deployment of RFID technology is likely to transform the healthcare sector.

Mutual authentication protocol for RFID conforming to EPC class 1 generation 2 standards. H. Chien and C. Chen, 2007. - proposed a new RFID authentication protocol conforming to EPC Class 1 Generation 2 standard. They claimed that this protocol is secure against DoS attack, replay attack, DATA forgery attack, and provides intractability and forward secrecy. In this paper, we show that the improved protocol has some weaknesses including DoS attack, back-end server impersonation, tag impersonation and DATA forgery attack.

LMAP: A real lightweight mutual authentication protocol for low-cost RFID tags.P. Peris-Lopez, J. Hernandez-Castro, J. Estevez-Tapiador, and A. Ribagorda, 2006- Low-cost Radio Frequency Identification (RFID) tags are devices of very limited computational capabilities, where only 250-3K logic gates can be devoted to security-related tasks. Many proposals have recently appeared, but all of them are based on RFID tags using classical cryptographic primitives such as PRNGs, hash functions, block ciphers, we propose a real lightweight mutual authentication protocol for low-cost RFID tags that offers an adequate security level.

\section{METHODOLOGIES}

In this project a for comma patient a Dynamic Service Non Dependency Verification has been implemented using IOT. The first process of this project initiate with hardware interface. We propose a heterogeneous IOT scheme to secure communication between a sensor node and an Internet host. We prove that this scheme is indistinguishable against various conditions. This project has the following advantages. First, it achieves confidentiality, integrity, authentication, and nonrepudiation in a logical single step. Second, it allows a sensor node in an identity-based Enhanced data transfer to send a message to an Internet host in a public key infrastructure method.

This project works under two phases

1) Offline phase

The hardware shows the corresponding sensor values in the hardware itself, without any internet connection. Using LCD display the values generated in the sensors will be displayed.

2) Online Phase

The phase need internet. Using COMM port from the hardware, the sensor value will be uploaded to a cloud server over internet. Multi user rights will be provided to view the sensor data from multiple locations and in multiple devices. 


\section{International Journal of Advanced Research in Computer and Communication Engineering}

Vol. 8, Issue 2, February 2019

Here we have some advantages, 1 . It can find the body movement of the coma patient and it can send the real time notification to doctors and relations of the coma patients. 2. The system can find the temperature variations like fever and can notify the doctors. 3. It can find the heart attack of the coma patients and it can alert the hospitals and as well as ambulance services 4 .low cost. 5. Low energy usage.

\subsection{ARDUINO UNO}

ARDUINO UNO is a microcontroller board based on the ATmega328P. It has 14 digital input/output pins (of which 6 can be used as PWM outputs), 6 analogy inputs, a $16 \mathrm{MHz}$ quartz crystal, a USB connection, a power jack, an ICSP header and a reset button. It contains everything needed to support the microcontroller; simply connect it to a computer with a USB cable or power it with a AC-to-DC adapter or battery to get started.

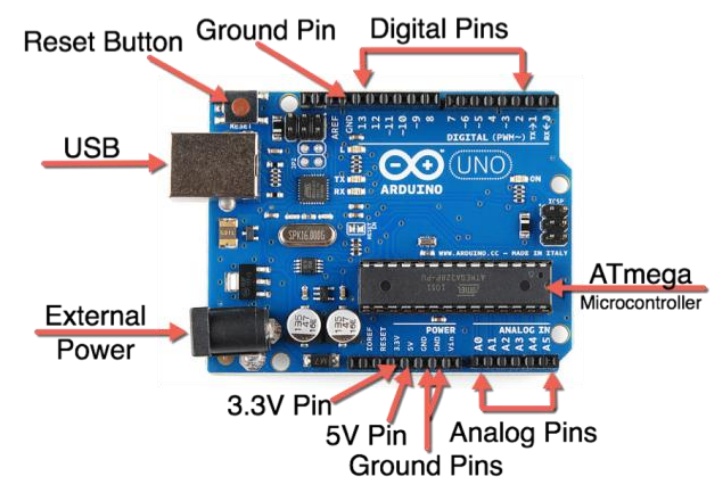

\subsection{TEMPERATURE SENSOR}

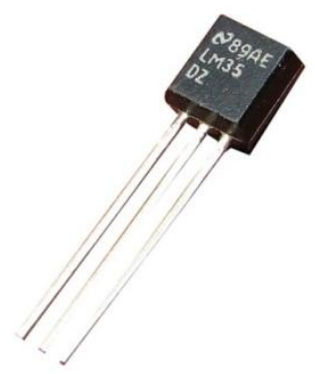

The LM35 series are precision integrated-circuit temperature devices with an output voltage linearly-proportional to the Centigrade temperature. The LM35 device has an advantage over linear temperature sensors calibrated in Kelvin, as the user is not required to subtract a large constant voltage from the output to obtain convenient Centigrade scaling. The LM35 device does not require any external calibration or trimming to provide typical accuracies of $\pm 1 / 4^{\circ} \mathrm{C}$ at room temperature and $\pm 3 / 4^{\circ}$ Cover a full $-55^{\circ} \mathrm{C}$ to $150^{\circ} \mathrm{C}$ temperature range. Lower cost is assured by trimming and calibration at the wafer level. The low-output impedance, linear output, and precise inherent calibration of the LM35 device makes interfacing to readout or control circuitry especially easy. The device is used with single power supplies, or with plus and minus supplies.

\subsection{PULSE SENSOR}

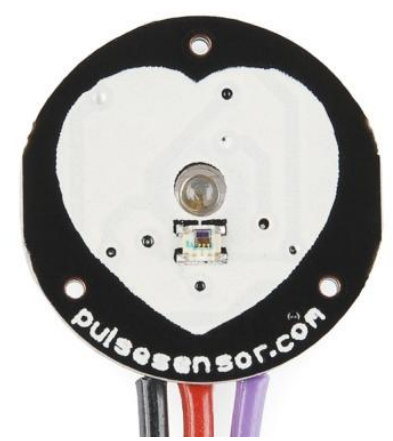

The working of the Pulse/Heart beat sensor is very simple. The sensor has two sides, on one side the LED is placed along with an ambient light sensor and on the other side we have some circuitry. This circuitry is responsible for the amplification 


\section{International Journal of Advanced Research in Computer and Communication Engineering}

Vol. 8, Issue 2, February 2019

and noise cancellation work. The LED on the front side of the sensor is placed over a vein in our human body. This can either be your Fingertip or you ear tips, but it should be placed directly on top of a vein. Using the pulse sensor is straight forward, but positioning it in the right way matters.

\subsection{FLEX SENSOR:}

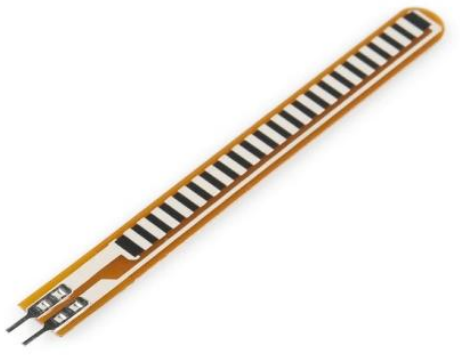

A simple flex sensor 2.2" in length. As you bend the sensor (flexing), the resistance across the sensor increases. The resistance of the flex sensor changes when the metal pads are on the outside of the bend (text on inside of bend). Connector is 0.1 " spaced and bread board friendly. Please refrain from flexing or straining this sensor at the base. The usable range of the sensor can be flexed without a problem, but care should be taken to minimize flexing outside of the usable range. For best results, securely mount the base and bottom portion and only allow the actual flex sensor to flex.

\subsection{GSM:}

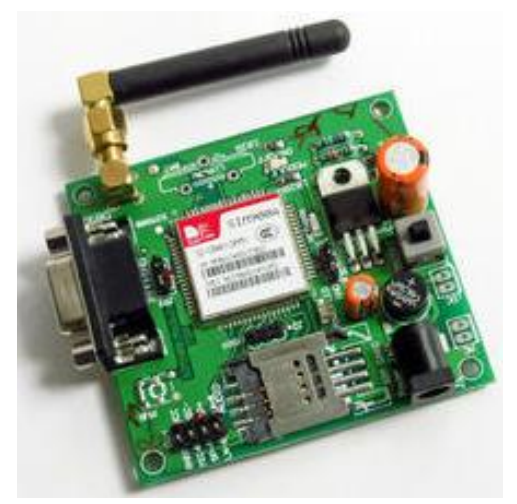

A GSM Module is basically a GSM Modem (like SIM 900) connected to a PCB with different types of output taken from the board - say TTL Output (for Arduino, 8051 and other microcontrollers) and RS232 Output to interface directly with a PC (personal computer). The board will also have pins or provisions to attach mic and speaker, to take out $+5 \mathrm{~V}$ or other values of power and ground connections. These types of provisions vary with different modules 3 .

\subsection{WIFI MODULE}

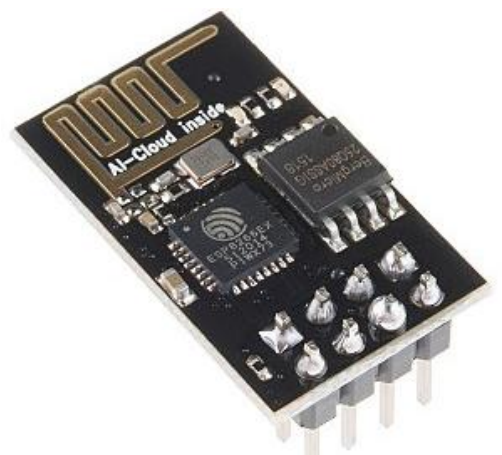

The ESP8266 WiFi Module is a self-contained SOC with integrated TCP/IP protocol stack that can give any microcontroller access to your WiFi network. The ESP8266 is capable of either hosting an application or offloading all Wi- 


\title{
International Journal of Advanced Research in Computer and Communication Engineering
}

\author{
Vol. 8, Issue 2, February 2019
}

Fi networking functions from another application processor. This module has a powerful enough on-board processing and storage capability that allows it to be integrated with the sensors and other application specific devices through its GPIOs with minimal development up-front and minimal loading during runtime.

\subsection{LCD DISPLAY}

We always use devices made up of Liquid Crystal Displays (LCDs) like computers, digital watches and also DVD and CD players. They have become very common and have taken a giant leap in the screen industry by clearly replacing the use of Cathode Ray Tubes (CRT). CRT draws more power than LCD and are also bigger and heavier. LCD's have made displays thinner than CRT's. Even while comparing the LCD screen to an LED screen, the power consumption is lesser as it works on the basic principle of blocking light rather than dissipating. All of us have seen an LCD, but no one knows the exact working of it. Let us take a look at the working of an LCD.

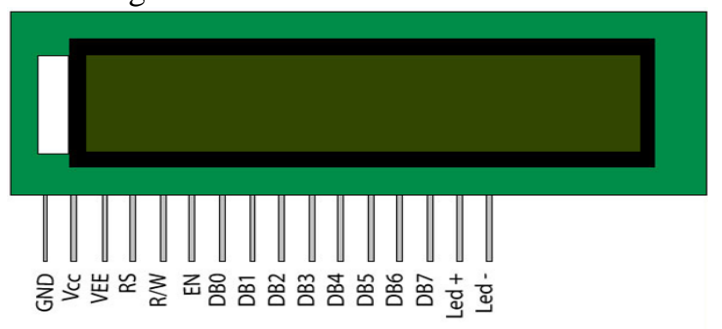

\subsection{POWER SUPPLY}

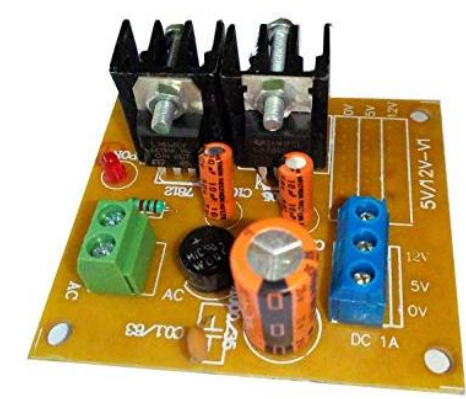

This is a simple approach to obtain a $12 \mathrm{~V}$ and 5V DC power supply using a single circuit. The circuit uses two ICs 7812 and 7805 for obtaining the required voltages. The AC mains voltage will be stepped down by the transformer, rectified by bridge and filtered by capacitor to obtain a steady DC level. The 7812 regulates this voltage to obtain a steady $12 \mathrm{~V}$ DC. The output of the IC1 will be regulated by the 7805 to obtain a steady $5 \mathrm{~V}$ DC at its output. In this way both $12 \mathrm{~V}$ and $5 \mathrm{~V}$ DC are obtained.

\section{CONCLUSION}

In this project we used a for comma patient a Dynamic Service Non Dependency Verification is implemented using IOT. The first process of this project initiate with hardware interface. And we used a heterogeneous IOT scheme to secure communication between a sensor node and an Internet host. We prove that this scheme is indistinguishable against various conditions. This project has the following advantages. First, it achieves confidentiality, integrity, authentication, and nonrepudiation in a logical single step. Second, it allows a sensor node in an identity-based Enhanced data transfer to send a message to an Internet host in a public key infrastructure method. Here we have some advantages, 1 . It can find the body movement of the coma patient and it can send the real time notification to doctors and relations of the coma patients. 2. The system can find the temperature variations like fever and can notify the doctors. 3. It can find the heart attack of the coma patients and it can alert the hospitals and as well as ambulance services 4. low cost. 5. Low energy usage

\section{REFERENCES}

[1]. A. M. Nia, M. Mozaffari-Kermani, S. Sur-Kolay, A. Raghunathan, and N. K. Jha, "Energy-efficient long-term continuous personal health monitoring," IEEE Trans. Multi-Scale Computing Systems, vol. 1, no. 2, pp. 85-98, 2015.

[2]. H. Ghayvat, J. Liu, S. C. Mukhopadhyay, and X. Gui, "Wellness sensor networks: A proposal and implementation for smart home for assisted living," IEEE Sensors Journal, vol. 15, no. 12, pp. 7341-7348, 2015.

[3]. S. C. Mukhopadhyay, "Wearable sensors for human activity monitoring: A review," IEEE Sensors Journal, vol. 15, no. 3, pp. 1321-1330, 2015. 


\title{
International Journal of Advanced Research in Computer and Communication Engineering
}

\author{
Vol. 8, Issue 2, February 2019
}

[4]. "Olympic medical institute validates Polar RS800 running computer and training system,” https://www.polar.com/usen/about polar/news/polar R800, accessed: 10-9-2016.

[5]. O. D. Lara and M. A. Labrador, "A survey on human activity recognition using wearable sensors," IEEE Communications Surveys \& Tutorials, vol. 15, no. 3, pp. 1192-1209, 2013.

[6]. W. Gao, S. Emaminejad, H. Y. Y. Nyein, S. Challa, K. Chen, A. Peck, H. M. Fahad, H. Ota, H. Shiraki, and D. Kiriya, "Fully integrated wearable sensor arrays for multiplexed in situ perspiration analysis," Nature, vol. 529, no. 7587, pp. 509-514, 2016.

[7]. A. Pantelopoulos and N. G. Bourbakis, "A survey on wearable sensor-based systems for health monitoring and prognosis," IEEE Trans. Systems, Man, and Cybernetics, vol. 40, no. 1, pp. 1-12, 2010.

[8]. S. Park, I. Locher, A. Savvides, M. B. Srivastava, A. Chen, R. Muntz, and S. Yuen, "Design of a wearable sensor badge for smart kindergarten," in Proc. IEEE Int. Symp. Wearable Computers, 2002, pp. 231-238.

[9]. A. Mosenia, S. Sur-Kolay, A. Raghunathan, and N. K. Jha, "CABA: Continuous authentication based on BioAura," IEEE Trans. Computers, DOI: 10.1109/TC.2016.2622262, 27 Oct., 2016.

[10]. A. B. Barreto, S. D. Scargle, and M. Adjouadi, “A practical EMGbased human-computer interface for users with motor disabilities," J. Rehabilitation Research and Development, vol. 37, no. 1, p. 53, 2000.

[11]. V. Pejovic, A. Mehrotra, and M. Musolesi, "Anticipatory mobile digital health: Towards personalised proactive therapies and prevention strategies," arXiv preprint arXiv:1508.03722, 2015.

[12]. W. Y. Wong and M. S. Wong, "Smart garment for trunk posture monitoring: A preliminary study," Scoliosis and Spinal Disorders, vol. 3, no. 1, p. 1, 2008.

[13]. A. Crane, S. Doppalapudi, J. O’Leary, P. Ozarek, and C. Wagner, "Wearable posture detection system,” in Proc. Annual Northeast Bioengineering Conference, 2014, pp. 1-2.

[14]. E. Sardini, M. Serpelloni, and M. Ometto, "Smart vest for posture monitoring in rehabilitation exercises," in Proc. IEEE Sensors Applications Symposium, 2012, pp. 1-5.

[15]. H. Harms, O. Amft, G. Tr “ oster, M. Appert, R. M “ uller, and A. Meyer-Heim, "Wearable therapist: Sensing garments for supporting children improve posture," in Proc. ACM Int. Conf. Ubiquitous Computing, 2009, pp. 85-88.

[16]. B. Ainsworth, L. Cahalin, M. Buman, and R. Ross, "The current state of physical activity assessment tools," Progress in Cardiovascular Diseases, vol. 57, no. 4, pp. 387-395, 2015.

[17]. M. de Zambotti, S. Claudatos, S. Inkelis, I. M. Colrain, and F. C. Baker, "Evaluation of a consumer fitness-tracking device to assess sleep in adults," Chronobiology International, vol. 32, no. 7, pp. 1024-1028, 2015.

[18]. J. Wei, "How wearables intersect with the Cloud and the Internet of Things: Considerations for the developers of wearables," IEEE Consumer Electronics Magazine, vol. 3, no. 3, pp. 53-56, 2014.

[19]. T. L. Koreshoff, T. Robertson, and T. W. Leong, "Internet of Things: A review of literature and products," in Proc. AMC Australian ComputerHuman Interaction Conference: Augmentation, Application, Innovation, Collaboration, 2013, pp. 335-344.

[20]. D. Malan, T. Fulford-Jones, M. Welsh, and S. Moulton, "CodeBlue: An ad hoc sensor network infrastructure for emergency medical care," in Proc. Int. Wkshp. Wearable and Implantable Body Sensor Networks, vol. 5, 2004.

[21]. V. Jones, A. van Halteren, N. Dokovsky, G. Koprinkov, R. Bults, D. Konstantas, and R. Herzog, "Mobihealth: Mobile health services based on body area networks," Centre for Telematics and Information Technology, Tech. Rep., 2006.

[22]. S. Ozcelik, "Drug infusion systems," Encyclopedia of Medical Devices and Instrumentation, pp. 495-508, 2006.

[23]. A. Hadjidj, M. Souil, A. Bouabdallah, Y. Challal, and H. Owen, "Wireless sensor networks for rehabilitation applications: Challenges and opportunities," J. Network and Computer Applications, vol. 36, no. 1, pp. 1-15, 2013.

[24]. A. Muro-de-la Herran, B. Garcia-Zapirain, and A. MendezZorrilla, "Gait analysis methods: An overview of wearable and non-wearable systems, highlighting clinical applications," Sensors, vol. 14, no. 2, pp. 3362-3394, 2014

[25]. W. Tao, T. Liu, R. Zheng, and H. Feng, "Gait analysis using wearable sensors," Sensors, vol. 12, no. 2, pp. 2255-2283, 2012. 